of great interest in connection with a case de- usually adults between the ages of thirty and scribed by Mallory. This case was one of a diffuse fifty. 7. Its progress is one of increasing severity. glioma in a child, which involved the meninges 8 . Medical treatment has little or no effect. 9. of the entire central nervous system. The Operations on peripheral branches give temporary macroscopic picture was that of a thick in- relief. Partial or complete removal of the flammatory exudation with more or less organization. The apparent origin was in the lumbar region. ${ }^{4}$

The possibility of a malignant diffuse glioma of this sort, limited to the meninges, arising from congenital nests, such as were found in our case, is certainly a very strong one.

\title{
OSMIC ACID INJECTIONS FOR THE RELIEF OF TRIFACIAL NEURALGIA.*
}

BY H. H. GERMAIN, M.D., Boston.

IN considering the subject of trifacial neuralgia one is struck by the diverse conditions said to be the cause of the disease. These cases may, I think, be divided roughly into three great classes.

1. Neuralgia, as a symptom of some general disease; anemia, diabetes, etc., where there are usually definite nerve changes; neuritis.

2. Reflex intermittent pain due to some local cause, as a carious tooth, sinus disease, etc.

3. True epileptiform neuralgia, to which neither true cause nor favoring conditions can be assigned.

It is the latter condition in which we are interested and which we are called upon to treat surgically sooner or later in its course.

Concerning the pathology of this condition, I desire to quote a few authorities showing that the pathological findings are by no means constant.

Spiller, who examined ganglia and nerves in 7 cases, describes degeneration of the myelin sheaths as well as endarteritis.

Keen considers that definite changes were demonstrated in 6 of the 7 cases.

Horsley has found thickening of the fibrous tissue sheath of the peripheral nerves. In general the Gasserian showed no definite changes.

Krause's observations on a series of cases were entirely negative.

John B. Murphy says that neuralgia is due to some cause which to date is unknown. He further states that the changes described by pathologists who have had the opportunity to make examinations of ganglia of trifacial neuralgia cases are almost as varied and diverse as the number of investigators.

Causes and symptoms. - The chief features of true major neuralgia are well summarized by Hutchinson, whom I quote as follows: 1. It is almost invariably unilateral. 2. It commences in the second or third division of the fifth nerve and tends to involve both. 3. The first division is involved to a much less degree. 4. Attacks of pain are paroxysmal or spasmodic and tend to increase in severity, with shortening intervals of freedom from pain. 5. During each attack there is usually spasm of the facial muscle of the affected side. 6. The subjects of the disease are

4 The Results of the Application of Special Histological Methods to the Study of Tumors. Sour. Exper. Med., September, 1908. * Read at a meeting of the Boston Society for Medical Improvement, Nov, 9, 1908.

This brings us to a surgical means which can be utilized for the relief of this disease. It has been common experience that nerve stretching, nerve section and avulsion of the whole nerve according to the method of Thiersch is followed by relief from pain for a longer or shorter period of time. All of these procedures are followed by regeneration of the peripheral nerve and recurrence of pain. The period of relief varies from three months to two years but is almost never permanent. Of late years certain injection methods have been in vogue, notably osmic acid injection and injections of alcohol.

About 1880 Neuber first used osmic acid for the relief of pain in a case of trifacial neuralgia, using a $1 \%$ solution. He injected the fluid in the region of the infra-orbital foramina. The relief was short lived and a second case was tried which remained well for three months. In these cases no incision was made nor did he attempt to inject the solution into nerve substance, as was done later by Bennet.

$J a c o b i^{1}$ reports 18 cases treated by injection of a $1 \%$ solution of osmic acid. He relieved 8 cases, 2 were improved and 8 not improved.

$W$. $H$. Bennet ${ }^{2}$ laid bare the nerves by an incision and injected 10 to $15 \mathrm{~mm}$. of a $1 \%$ solution into the nerve trunk. His results quoted by Murphy are as follows: two patients well at the end of four years; 2 patients well at the end of three years; 3 patients well at the end of two years; 2 patients well at the end of one year; 2 recurred early (three and six months); 2 died of some other cause without a recurrence (six and nine months after operation); 6 cases were lost sight of.

J. B. Murphy ${ }^{3}$ reports 14 cases with favorable immediate results. $\mathrm{He}$ injects a $2 \%$ solution directly into the nerve trunk.

I have operated on 11 cases by this method as follows:

CASE I. F., age, fifty. Neuralgia of third divisions of the fifth nerve of one year's duration; takes about two grains of morphia daily. Infra-orbital, inferior dental and lingual nerves exposed and injected with a $2 \%$ solution of osmic acid. Complete relief from pain after thirty-six hours. Reported well two years later.

CASE II. F., age, fifty-two. Neuralgia of first and second divisions of six months' duration; takes both morphine and cocaine daily. First and second divisions exposed and injected as above. Relief for two months, after which she relapsed and took morphia habitually.

CASE III. F., age, sixty-five. Neuralgia of inferior dental nerve, two years' duration. Nerve exposed and in jected in bony canal and at mental foramen. Reported well two and a half years after operation.

CASE IV. M., age, seventy. Neuralgia of second and

1 Jancet, 1885

Ibid., Nov. 4,1899

3 Jour. Am. Med. Asso., 1904. 
third divisions of the fifth of one year's duration. Nerves exposed and injected as above. Relief from pain for three months followed by recurrence in the lingual nerve. This subsided and the patient was reported well six months after operation. He was then lost sight of.

CAsE V. F., age, sixty-five. Neuralgia of third division of the fifth of two months' duration. Lingual and inferior dental nerve exposed and injected as above with complete relief thrce years after.

CASE VI. M., age, forty-two. Neuralgia of first division of fifth nerve exposed in orbit, and supraorbital and supra-trochlear divisions injected as above. Recurrence two years afterwards.

Case VII. F., age, thirty-seven. Neuralgia of third division of the fifth. Inferior dental and lingual exposed and injected. Injection followed by cellulitis of the mouth, necessitating an incision. Relief for one year. Second operation revealed nerve surrounded with scar tissue. Nerve avulsed. Recurrence in eight months. Third operation, avulsion of third division of the fifth at the foramen ovale. Relief to date; one month after operation.

Case VIII. M., age, thirty-five. Neuralgia first division of the fifth of three months' duration. Had been treated for a supposed suppuration of frontal sinus. Supra-orbital nerve exposed and injected as above. Relief one and a half years after operation.

CASE IX. M., age, thirty-eight. Neuralgia of the right supra-orbital, one year's duration. Nerve exposed and injected with a $2 \%$ solution. Partial relief from pain in twenty-four hours and complete absence from pain in three days. Reported well two years after operation.

Case X. F., age, seventy. Neuralgia of the inferior dental and infra-orbital nerve. Recurrent attacks of pain for ten years with a few weeks' interval. Infra-orbital nerve exposed and injected with a $2 \%$ solution. Complete relief after six days. Reported well sixteen months after.

CASE XI. F., age, forty. Neuralgia in infra-orbital and infra-dental regions. Intervals two years. Infraorbital injected on both sides. No recurrence eighteen months after in the infra-orbital, but recurrence in the infra-dental which was not infected.

Results are summarized as follows: 3 cases well two years after; 1 case well three years after; 2 cases well one and one-half years after; 1 case well sixteen months after but with recurrence in another branch; 1 recurrence in two months; 1 recurrence in one year; 1 recurrence in two years; 1 case lost sight of.

From my personal experience which has been confined to these few cases I conclude that osmic acid injections will relieve trifacial neuralgia for a longer or a shorter period of time. 2. That relief from pain is not immediate but follows in a few days after injection. 3. That it may be followed by a certain amount of necrosis of tissue at the point of injection. 4. That it is little if any better than other peripheral operations. 5 . It is best used in a $2 \%$ solution injected directly in to the nerve, using a glass syringe and a platinum needle. 6 . It is to be used only in purely sensory nerves as its employment in mixed nerves is followed by motor paralysis.

IT is announced that Dr. Charles S. Minot of the Harvard Medical School, has been appointed a foreign corresponding member of the Académie Royale de Médicine de Belgique.

\section{ghedical Proureg.}

\author{
REPORT ON OBSTETRICS.
}

Ophthalmia Neonatorum.

BY ROBERT L. DE NORMANDIE, M.D., BOSTON.

"SHOULd one or both eyes of an infant become inflamed, swollen and red and show an unnatural discharge at any time within two weeks after birth, it shall be the duty of the nurse, relative or other attendant having charge of such infant to report in writing within six hours thereafter to the board of health of a city or town in which the parents of the infant reside the fact that such inflammation, swelling and redness of the eyes and unnatural discharge exist. On receipt of such report or notice of the same symptoms given by a physician as provided by the following section, the board of health shall take such immediate action as it may deem necessary in order that blindness may be prevented. Whoever violates the provision of this section shall be punished by a fine of not more than $\$ 100$.

"If a physician knows that a person whom he is called to visit is infected with smallpox, diphtheria, scarlet fever or any other disease declared by the State Board of Health to be dangerous to the public health, or if one or both eyes of an infant whom or whose mother he is called to visit become inflamed, swollen or red and show an unnatural discharge within two weeks after the birth of such infant, he shall immediately give notice thereof in writing over his own signature to the selectmen or board of health of the town, and if he refuses or neglects to give such notice, he shall forfeit not less than $\$ 50$ nor more than $\$ 200$ for each offense."

The above law was passed by the Massachusetts legislature in the session of 1905 and is now upon the statute books. Relatively few physicians even know of it in the above-quoted form. They know they must report smallpox, scarlet fever, etc., but that there is any such broad, inclusive, far-reaching law in regard to ophthalmia neonatorum I venture to say that the great majority of them do not. It is because of this law, because of the importance of prophylaxis and early treatment, that I write upon this subject. The reduction of blindness in the infant lies largely in a proper prophylaxis at birth, and upon early, efficient and well-directed treatment if ophthalmia develops. The physician present at the birth is the one on whom the responsibility rests in by far the greater number of cases, and when he realizes that for his neglect in reporting a case of ophthalmia there is a statute which provides that "he shall forfeit not less than $\$ 50$ nor more than $\$ 200$ for each offense " it seems to me that the cases of ophthalmia will become much fewer; surely the neglected cases where blindness ensues will be materially reduced.

By ophthalmia neonatorum the profession has come to understand an infection of one or both eyes of the infant, occurring within the first few days of life, caused by the diplococcus of Neisser, 